\title{
Simulation of Wind Loads on High Rise Buildings Taking into Account the Construction Site Environment
}

\author{
Aleksei.A. Antipin, V.N. Alekhin, S.N. Gorodilov, S.V. Khramtsov , A.S. Noskov \\ Ural Federal University named after the first President of Russia B.N. Yeltsin, Ekaterinburg, Russia \\ e-mail: sapros_ustu@mail.ru
}

\begin{abstract}
The paper presents the results of numerical simulation of wind pressure on high rise buildings being under construction and planned in Yekaterinburg city (Russia). Simulation is performed in the program ANSYS. The simulated building is placed in a domain that is the numerical analogue of wind tunnel. Domain sizes are chosen in such a way that simulated buildings do not affect the flow of air on its boundaries. Shear stress transport (SST) turbulence model is used. In order to validate the numerical model the paper presents the results of experimental study and numerical simulation of external air flow around the cylindrical body with the diameter of $8 \mathrm{~mm}$, placed on the way of free submerged jet of air. The cylinder which has simple geometry form was used due to the need to achieve the generality of the outcomes. A comparison of the results of calculation of wind pressure with the experimental data blasting building models in wind tunnels is done.
\end{abstract}

Keywords-component: high-rise building, wind impact, simulation, wind loads.

\section{INTRODUCTION}

Wind loads on high-rise buildings are referred to the basic load group. Most design works considerably deal with aerodynamic analysis. Expert assessment of the standard values of wind pressures for buildings higher than 200 meters (corresponding to wind region VI, Russia) shows that the wind pressure can be more dangerous to the overall strength than the nine-point earthquake. Civil Engineering Institute of Ural Federal University named after the first President of Russia B. N. Eltsin steadily deals with design and deformation monitoring of a number of high-rise buildings in Ekaterinburg [1, 2]. Both wind pressure calculation and blow-through in wind tunnels of the building models were performed. Numerical and experimental data convergence was found to be satisfactory (within $10-30 \%$ ).

\section{COMPUTATIONAL MODEL}

Numerical simulation is performed using finite element method applied in the software package ANSYS. The computational model is the numerical analogue of wind tunnel. The high-rise buildings and surrounding objects are placed in the domain, whose sizes are selected so that air flow on its boundaries is not affected by the buildings placed in it. The example of such domain is shown in Fig.1. A simulated high-rise building is on the left; the domain with the building proper and surrounding objects in it is on the right.

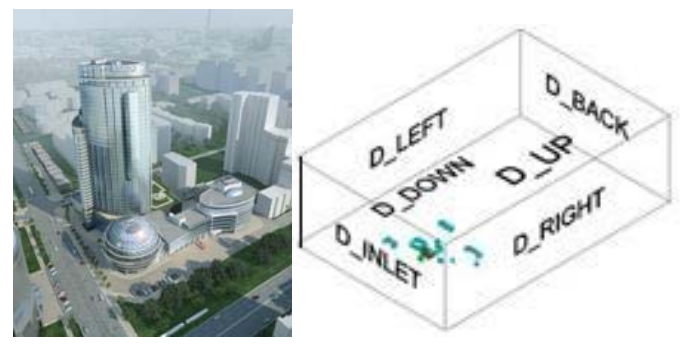

Figure 1. Simulated high-rise building and domain

The size of the computational domain in vertical, lateral and longitudinal flow directions is conditioned by the simulated site development and boundary conditions. In the experience of testing in wind tunnels the building height $\mathrm{H}$ is assumed to affect up to a distance of $10 \mathrm{H}$. This height can be recommended to be an essential requirement to the model. According to test calculations made by the Institute of Architecture AIJ, Japan [3], the size of the computational domain in the vertical flow direction for an isolated building should be not less than $5 \mathrm{H}$.

\section{DISCUSSION}

Analysis of wind pressure calculations proves the problem of data accuracy to be unsolved. Paper [6] shows that one fails to consider the essential parameters like scale factor, rigidity parameter, etc. while testing high-rise building models in wind tunnels. In addition, the agreement between experimental data and actual pressure values remains vague in computational analysis.

Diverse semi-empirical turbulence models are currently developed $[5,6]$. Meanwhile each model is acceptable only in a limited number of simulation cases. This resulted from significant differences in turbulence internal structure arising from varied fluid flow conditions. A unified turbulence model valid for all feasible simulation cases has not been developed yet. Two parameter semi-empirical turbulence models (k- $\varepsilon, \mathrm{k}-\omega$ etc.) are mainly practiced [6]. The given models enable relatively simple flows (e.g. fluid flow in a pipeline) to be predicted with an adequate accuracy but in the simulation of more complicated cases they provide qualitatively and quantitatively inadequate results [7].

For outward fluid flow near-wall boundary layers are essential as Reynolds numbers change in a wide range and 
partial flow laminarization may arise there. For example, in the flow past a cylindrical body one can observe several fluid flow conditions: the boundary cylinder layer is laminar when Reynolds numbers are less than a critical number $\left(\operatorname{Re}<\mathrm{Re}_{\mathrm{cr}}\right)$. Separation of laminar boundary layer occurs in the frontal side of a cylinder with separation angle $\varphi=82^{\circ}$ [8]. Thus, proper mathematical formulation of fluid laminarization effects in near-wall layers is of great importance. To this end, authors [6] designed and proposed the transition turbulence model to be applied in the case of significant Reynolds number decreasing. The application of the suggested model along with the model of turbulence SST allows to predict more accurately fluid behavior in a boundary layer in various fluid conditions both subcritical and supercritical.

Numerical simulation and experimental determination of wind velocities when flowing past a cylindrical body were carried out in order to estimate the accuracy of resulting values. The research task was testing and verification with respect to experimental measuring results of varied semi-empirical turbulence models in the case of outward flow past a cylindrical body placed in the passage of a freely submerged air stream.

The main model SST ratios are given below.

The equation for turbulent kinetic energy $\mathrm{k}$ and turbulence frequency $\omega$ :

$$
\begin{aligned}
& \frac{\partial \rho k}{\partial t}+\frac{\partial \rho \bar{U}_{j} k}{\partial x_{j}}=P_{k}-\beta^{*} \rho \omega k+\frac{\partial}{\partial x_{j}}\left(\Gamma_{k} \frac{\partial k}{\partial x_{j}}\right) \\
& \frac{\partial \rho \omega}{\partial t}+\frac{\partial \rho \bar{U}_{j} \omega}{\partial x_{j}}=\frac{\alpha}{v_{t}} P_{k}-\beta^{*} \rho \omega k+ \\
& +\beta \rho \omega^{2} \frac{\partial}{\partial x_{j}}\left(\Gamma_{\omega} \frac{\partial \omega}{\partial x_{j}}\right)+\left(1-F_{1}\right) 2 \rho \sigma_{\omega 2} \frac{1}{\omega} \frac{\partial k}{\partial x_{j}} \frac{\partial \omega^{\prime}}{\partial x_{j}}
\end{aligned}
$$

Where $\rho$-density; $\mathrm{y}$ - the distance to the surface; $\bar{U}_{j}$ flow velocity.

$$
\begin{aligned}
& P_{k}=\min \left(\mu_{t} S^{2}, 10 \beta^{*} \rho \omega k\right) \\
& \Gamma_{k}=\mu+\frac{\mu_{t}}{\sigma_{k}}, \Gamma_{\omega}=\mu+\frac{\mu_{t}}{\sigma_{\omega}}, S=\sqrt{2 S_{i j} S_{j i}} \\
& \sigma_{k}=\frac{1}{\frac{F_{1}}{\sigma_{k 1}}+\frac{\left(1-F_{1}\right)}{\sigma_{k 2}}}, \sigma_{\omega}=\frac{1}{\frac{F_{1}}{\sigma_{\omega 1}}+\frac{\left(1-F_{1}\right)}{\sigma_{\omega 2}}}, \\
& \sigma_{k 1}=1,176 ; \sigma_{k 2}=1 ; \sigma_{\omega 1}=2 ; \sigma_{\omega 2}=1,168 \\
& v_{t}=\frac{a_{1}}{\max \left(a_{1} \omega, S F_{2}\right)}-\text { turbulent viscosity; } \\
& \alpha=\alpha_{1} F_{1}+\alpha_{2}\left(1-F_{2}\right) \\
& \arg _{1}=\min \left(\max \left(\frac{\sqrt{k}}{\beta^{*} \omega y} ; \frac{500 \mu}{\rho y^{2} \omega}\right) ; \frac{4 \rho \sigma_{\omega, 2} k}{C D_{k \omega} y^{2}}\right)
\end{aligned}
$$

The positive part of transverse diffusion terms is calculated according to:

$$
C D_{k \omega}=\max \left(2 \rho \sigma_{\omega, 2} \frac{1}{\omega} \frac{\partial k}{\partial x_{j}} \frac{\partial \omega}{\partial x_{j}} ; 10^{-10}\right)
$$

Turbulence viscosity is calculated by the equation:

$$
\mu_{t}=\min \left[\frac{p k}{\omega} ; \frac{a_{l} p k}{S F_{2}}\right] \text {, with constant } a_{l}=0.31 \text { and }
$$

blending function.

$$
F_{2}=\tanh \left(\arg _{2}^{2}\right) \text {, }
$$

Where $\arg _{2}=\left[\max \left(\frac{2 \sqrt{k}}{\beta^{*} \omega y} ; \frac{500 v}{y^{2} \omega}\right)\right]^{2}$

Blending functions $F_{1}$ и $F_{2}$ are given so that to define whether the point in question is inside or outside the surface boundary layer.

Consequently, functions $F_{1}$ and $F_{2}$ possess the following limit values:

$$
F_{1}=0 \text {, off the surface } \Rightarrow k-\varepsilon \text { model }
$$
model

$F_{1}=1$, inside the boundary layer at surfaces $\Rightarrow k-\omega$

$$
\begin{aligned}
& F_{2}=0 \Rightarrow \text { combination } k-\varepsilon \text { and } \Rightarrow k-\omega \\
& F_{2}=1 \Rightarrow \text { SST model }
\end{aligned}
$$

\section{EXPERIMENTAL UNIT}

Most researchers are known to devote their work to this problem [6]. The following scheme of an experimental unit was chosen on the basis of their research results (Fig. 2).

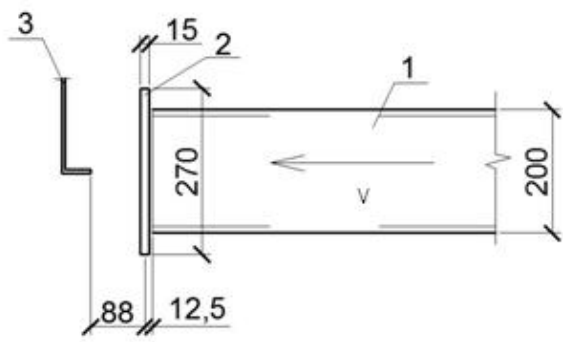

Figure 2. Scheme of an experimental unit

Wind from nozzle 1 flows over a round cylinder 2 . Prandtl tube 3 fixed on the measuring plane is placed at a distance of $88 \mathrm{~mm}$ from the cylinder centre 2. Measurements are done with coordinate spacer changing its position on the horizontal axis "Y" every $5 \mathrm{~mm}$ and vertical axis " $Z$ " every $10 \mathrm{~mm}$ respectively. Fig. 3 shows axis designation adopted in the paper. 


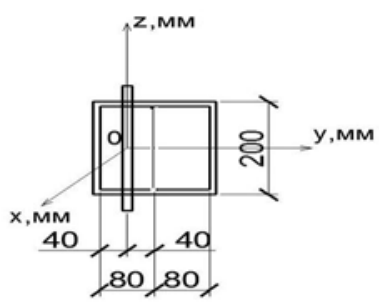

Figure 3. Axis designation

\section{COMPuter Model of A CYLINDER}

Tetrahedral finite element mesh was used. Calculations were done for several mesh options with varied finite element dimensions (Fig. 4). Figure 5 shows the computational domain.

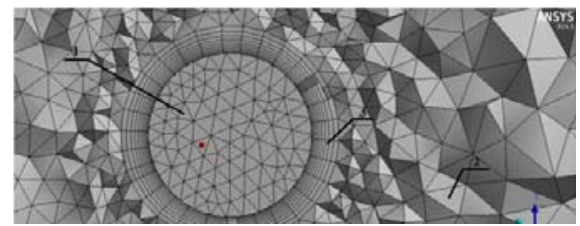

Figure 4. Mesh around the cylinder

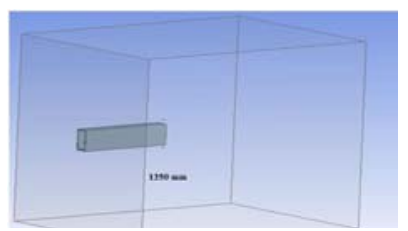

Figure 5. The computational domain.

\section{RESULTS}

The best numerical analysis results was obtained for the following mesh parameters: cell dimensions of the mesh ( $\mathbf{m m})$ - cylinder -0.3 , domain -3 ; number of boundary layers - 5; boundary layer thickness $(\mathrm{mm})-0.1$; enlargement factor -1.2 .

Figure 6 shows the comparison of experimental data of wind velocities for 6 turbulence models. Thus, the results being in agreement with experimental data can be obtained by means of the model SST.

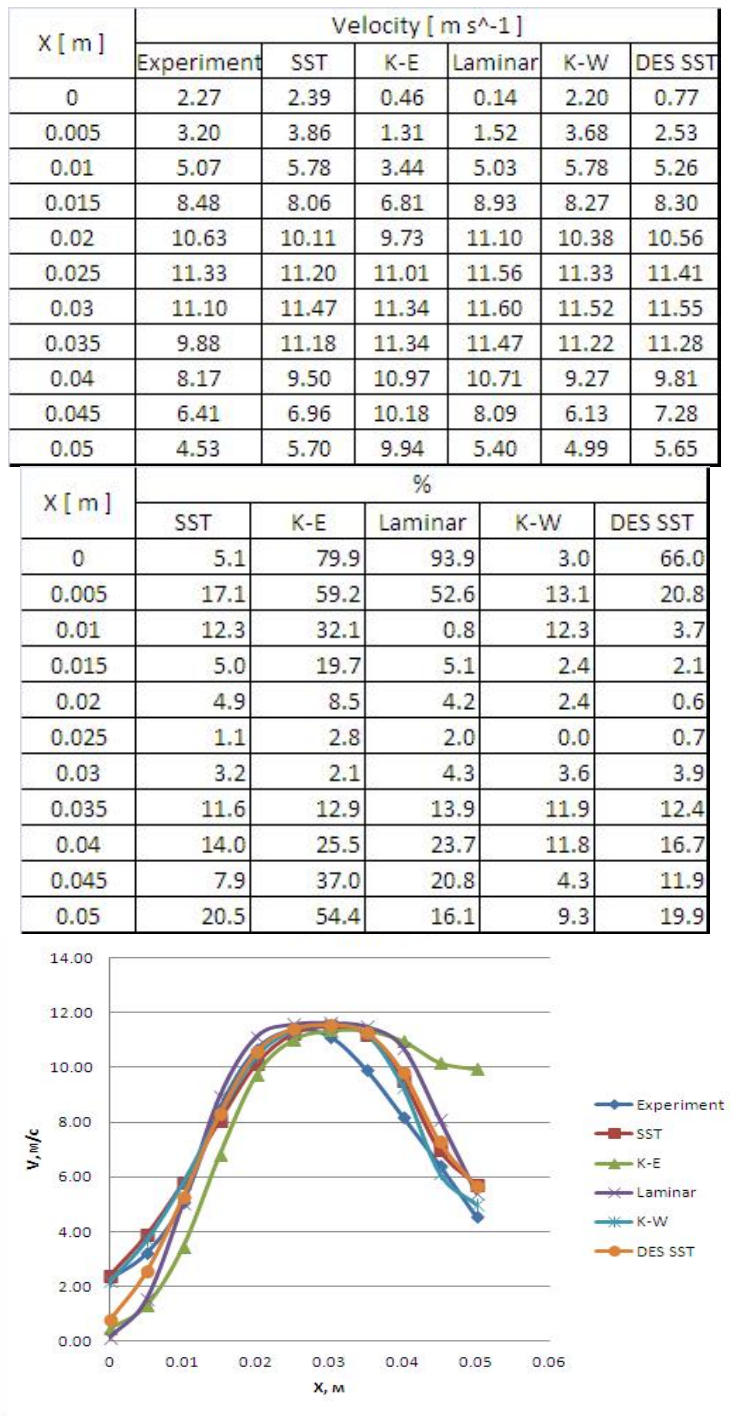

Figure 6. Comparison of wind velocities.

\section{COMPARISON OF EXPERIMENTAL DATA} WITH NUMERICAL ANALYSIS

The comparison of calculated and experimental data is performed by the example of multifunctional business center in Yekaterinburg (Figure 7). Buildings, included in a domain are shown in Figures 8 and 9. The multifunctional business center is highlighted in green. 


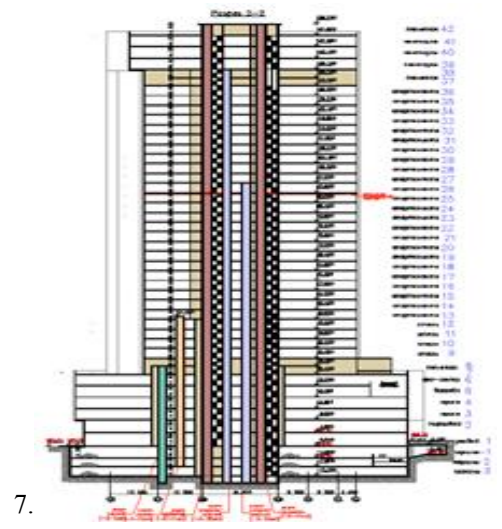

Figure 7. Multifunctional business center (project).

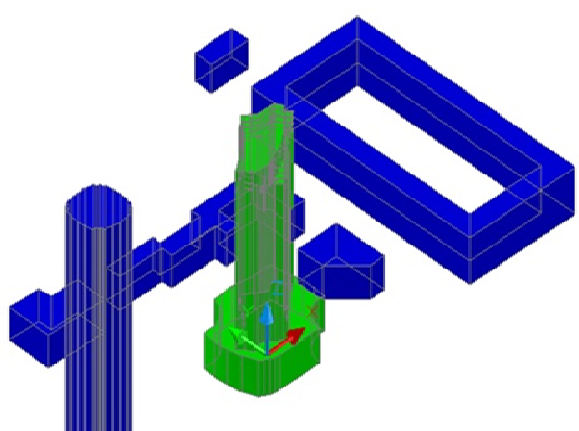

Multifunctional business centre

Figure 8. Buildings, included in the domain.

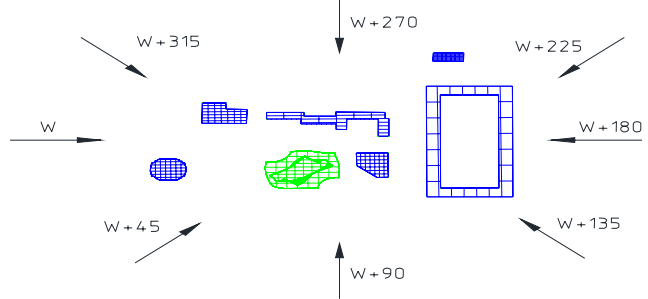

Figure 9. Buildings, included in the domain.

Comparison of experimental and analysis results are presented as follows. The outer contour of the building is divided into sections shown on figure 10. The sections of computer-model are shown on the left, and - an experimental model on the right.

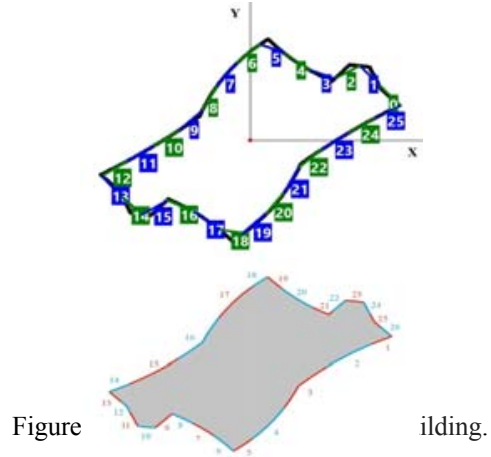

Figure 10. The outer contour of the building.

As can be seen in Figure 11 the results of numerical analysis and experimental wind determination are qualitatively similar. The difference of computational and experimental values is mainly due to varied comutational models of buildings used in natural oscillination frequency tests. Maximum wind loads obtained numerically are in good agreement with those obtained experimentally.
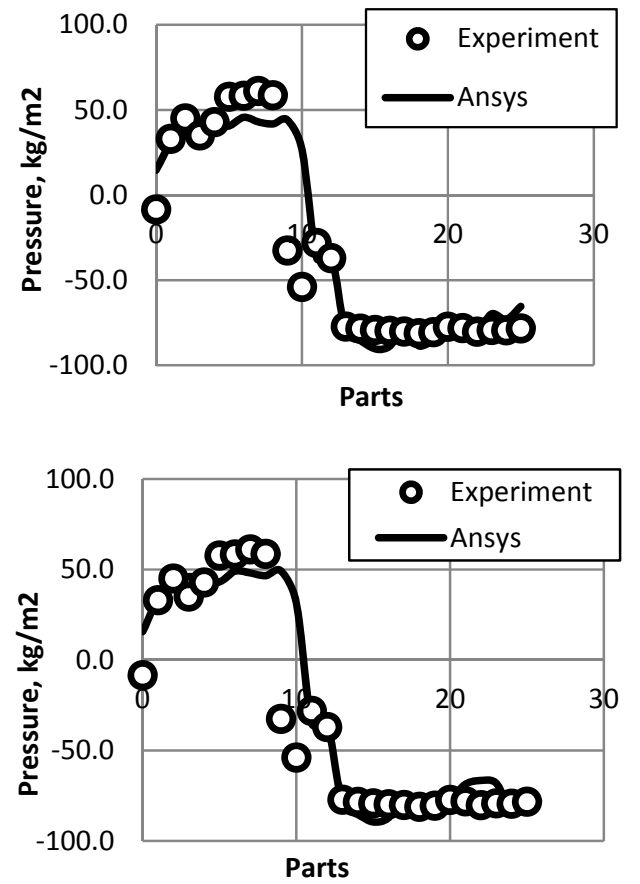

Figure 11 Comparison of numerical and experimental results. 


\section{CONCLUSIONS}

Comparison of numerical and experimental results shows, that developed technique of numerical simulation can be used to determine the wind pressure on high-rise buildings.

\section{REFERENCES}

[1] V.N. Alekhin, A.A. Antipin, S.N. Gorodilov. Analysis of wind impacts on the high-rise building "Iset Tower. Applied Mechanics and Materials. 2013 281. 639-644.

[2] Vladimir Alekhin, Aleksey Antipin, Sergey Gorodilov, Sergey Khramtsov. Numerical simulation of wind loads on high rise buildings. Proceedings of 13th International Conference on Construction Applications of Virtual Reality.-London:2013. 2013. 620-628.
[3] AIJ Recommendations for Loads on Buildings. // Architectural Institute of Japan, Japan. 1996.

[4] J. Wacker, R. Friedrich, E.J. Plate, U. Bergdolt. Fluctuating wind load on cladding elements and roof pavers. J. of Wind Engineering and Industrial Aerodynamics, 38, 1991, p. 405-418.

[5] P.G. Frik. Turbulence: models and approaches. Perm, Russia, 1998.

[6 David C. Wilcox. Turbulence modeling for CFD, DCW Industries, California, 1994. $460 \mathrm{p}$

[7] F.R. Menter. Review of the shear-stress transport turbulence model experience from an industrial perspective. International Journal of Computational Fluid Dynamics, Vol. 23 No. 3, 2009. P. 305 - 316.

[8] H. Schlichting. Boundary layer theory. Translation from German into Russian.

Moscow,

1974

712 